\title{
Titer and Product Affect the Distribution of Gene Expression after Intraputaminal Convection-Enhanced Delivery
}

\author{
Marina E. Emborg ${ }^{a-c}$ Samuel A. Hurley Valerie Joers $^{a, b}$ Do P.M. Tromp ${ }^{b, d, e}$ \\ Christine R. Swanson $^{a}{ }^{b}$ Sachiko Ohshima-Hosoyama ${ }^{a}$ Viktorya Bondarenko $^{a}$ \\ Kyle Cummisford $^{a}$ Marc Sonnemans ${ }^{f}$ Stephan Hermening ${ }^{f}$ Bas Blits ${ }^{f}$ \\ Andrew L. Alexander ${ }^{\text {b-e }}$ \\ a Preclinical Parkinson's Research Program, Wisconsin National Primate Research Center, ${ }^{b}$ Neuroscience Training

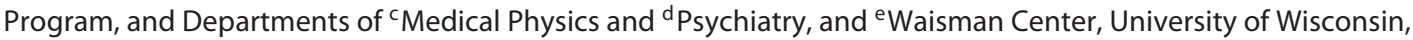 \\ Madison, Wis., USA; ${ }^{f}$ uniQure BV, Amsterdam, The Netherlands
}

\section{Key Words}

Convection-enhanced delivery - Gene expression .

Intracerebral brain therapy · Parkinson's disease

\begin{abstract}
Background: The efficacy and safety of intracerebral gene therapy for brain disorders like Parkinson's disease depends on the appropriate distribution of gene expression. Objectives: To assess whether the distribution of gene expression is affected by vector titer and protein type. Methods: Four adult macaque monkeys seronegative for adeno-associated virus 5 (AAV5) received a 30- $\mu$ l inoculation of a high- or a lowtiter suspension of AAV5 encoding glial cell line-derived neurotrophic factor (GDNF) or green fluorescent protein (GFP) in the right and left ventral postcommissural putamen. The inoculations were conducted using convection-enhanced delivery and intraoperative MRI (IMRI). Results: IMRI confirmed targeting and infusion cloud irradiation from the catheter tip into the surrounding area. A postmortem analysis 6 weeks after surgery revealed GFP and GDNF expression ipsilateral to the injection site that had a titer-dependent dis-
\end{abstract}

\section{KARGER}

E-Mail karger@karger.com

www.karger.com/sfn tribution. GFP and GDNF expression was also observed in fibers in the substantia nigra (SN) pars reticulata (pr), demonstrating anterograde transport. Few GFP-positive neurons were present in the SN pars compacta (pc), possibly by direct retrograde transport of the vector. GDNF was present in many neurons of the SNpc and SNpr. Conclusions: After controlling for target and infusate volume, the intracerebral distribution of the gene product was affected by the vector titer and product biology.

(c) 2014 S. Karger AG, Basel

\section{Introduction}

The usage of convection-enhanced delivery (CED) for intracerebral dosing of therapeutic molecules is intended as a method of maximizing the distribution per inoculation [1] while minimizing the risks associated with multiple injection sites [2]. CED, combined with intraoperative MRI (IMRI), increases targeting accuracy and allows monitoring the infusion cloud [3-5]. These new technologies are particularly attractive for intracerebral gene 
therapy as the efficacy and safety of this approach depends on an appropriate vector distribution and, ultimately, gene expression.

In the last decade, adeno-associated virus (AAV) serotype 2 (AAV2) has been the vector of choice for preclini$\mathrm{cal}$ and clinical research into the central nervous system. More recently, other serotypes have been characterized for their different cellular affinity and transfection efficacy [6]. From these investigations, AAV5 has emerged as an alternative candidate for brain gene therapy applications. AAV5 seems to have a more widespread distribution after intracerebral inoculation and to be more neurotropic than AAV2 [6]. In humans, the positive AAV5 serotype seems to be less frequent than the AAV2 serotype, which can be an advantage for long-term treatments as circulating antibodies against the vector may inhibit gene transfer [7].

The intracerebral distribution of any injected solution is affected by physical factors such as the characteristics of the infusion catheter, infusion volume and rate. The anatomical target, which may have natural boundaries and/or outward paths for fluid flow, can also affect the infusate distribution [4]. Investigators have proposed that the gene expression of molecules like glial cell linederived neurotrophic factor (GDNF) can be predicted by monitoring the infusion cloud using IMRI and gadoteridol coinfusions [8]. Yet, the molecular weight of the infusate and biological characteristics also affect intracerebral spread, which is further complicated when considering gene therapy approaches. In gene therapy, a suspension containing viral vectors is injected to produce the gene of interest. Therefore, the allocation of the therapeutic molecule can be affected by viral vector factors (e.g. serotype and infused titer) as well as by the characteristics of the gene product (e.g. whether the produced molecule is released or remains intracellular).

In this report, we aimed to assess the gene expression pattern associated with the vector titer and protein type. We hypothesized that: (1) inoculation of a higher vector titer would produce a greater spread than a lower titer and (2) GDNF, a protein excreted by cells, would be able to spread farther than green fluorescent protein (GFP), which remains intracellular after synthesis. Nonhuman primates received intracerebral CED of AAV5 encoding for GDNF or GFP. The vector was produced using a baculovirus-mediated platform that is stable, scalable, validated and cGMP-compliant, which is necessary for possible future clinical application. Due to our interest in Parkinson's disease (PD), we targeted the postcommissural putamen nucleus. This brain area receives dopami- nergic terminals from the substantia nigra (SN) pars compacta (pc) and is severely affected by PD. In clinical trials, the putamen nucleus has been targeted for trophic factor delivery by direct protein infusion (e.g. Morrison et al. [9]) or by gene therapy (e.g. Marks et al. [10]). GDNF has dopaminotrophic properties and has been proposed as a candidate for trophic therapy to slow down PD progression [9].

\section{Materials and Methods}

\section{Animals}

This study was performed in strict accordance with the recommendations set up in the Guide for the Care and Use of Laboratory Animals by the National Institutes of Health (1996) in a facility accredited by the Association for Assessment and Accreditation of Laboratory Animal Care (Wisconsin National Primate Research Center, University of Wisconsin, Madison, Wis., USA). The experimental protocol was approved by the Institutional Animal Care and Use Committee at the University of Wisconsin (permit No. G00628). All efforts were made to minimize the number of animals used and to ameliorate any distress.

Four male adult rhesus monkeys (Macaca mulatta; age: 5-10 years; weight: 7-15 kg) seronegative for AAV5 were used for this project. The animals were housed individually on a 12-hour light/ dark cycle and they received food and water ad libitum. The animals' diet was supplemented with fruit and vegetables. General health monitoring included weight, food intake, blood chemistry, coagulation and urinalysis.

\section{Vector Preparation}

The AAV vector encoding for GDNF or GFP was packaged into AAV5 at facilities of Amsterdam Molecular Therapeutics (nowadays uniQure BV) and produced according their dedicated procedures. The therapeutic expression cassette of AAV5 contains the cDNA of the human GDNF gene, isoform 1, which is the longest isoform of $636 \mathrm{bp}$, coding for the pre-pro form. Expression is under the control of the CAG promoter, a combination of the cytomegalovirus early enhancer element and chicken $\beta$-actin promoter. GDNF is preceded by a Kozak sequence and polyadenylated by the bovine growth hormone polyadenylation signal. The whole cassette is flanked by two noncoding inverted terminal repeats of AAV2. As a control vector, the gene encoding the GDNF cDNA was substituted for the enhanced GFP cDNA.

Recombinant AAV5 vectors were prepared using a baculovirus expression system, as described earlier $[11,12]$. Briefly, three recombinant baculoviruses - one encoding for REP for replication and packaging, one encoding for CAP-5 for the capsid of AAV5 and one with the expression cassette - were used to infect $\mathrm{Sf} 9$ insect cells. Purification was performed using AVB Sepharose high-performance affinity medium (GE Healthcare, Piscataway, N.J., USA). The vectors were titered using a quantitative PCR (qPCR) method with primer-probe combinations directed against the transgene, and titers were expressed as genomic copies (GC) per milliliter. The titers were in the range of $1-3^{13} \mathrm{GC} / \mathrm{ml}$ for both vectors and then diluted to planned concentrations. 


\section{AAV5 Intracerebral Infusions}

Bilateral viral vector infusions by CED into the ventral postcommissural putamen were performed using IMRI guidance and a pivot point-based MRI-compatible external trajectory guide, as previously described $[3,4]$. Briefly, placement of the system base was performed under sterile conditions and isoflurane (1-3\%) anesthesia using MRI-guided stereotactic methods in a state-ofthe-art surgical suite adjacent to the MRI suite. An animal was placed in an MRI-compatible stereotactic frame following coordinates similar to those used for the baseline MRI. The animal's vital signs were monitored throughout the procedures. Two 6-mm-diameter craniotomies were drilled into the planned entry areas, corresponding to the opening of the trajectory guide base, and the dura was retracted to expose the brain. The bases were mounted to the skull over the craniotomy with 3 small self-tapping screws.

For the final targeting planning and intracerebral introduction of the catheter, the monkey was transported from the surgical suite to the MRI suite while placed in the stereotactic frame and receiving isoflurane anesthesia. A cannula filled with sterile degassed water was inserted into the stem of the trajectory guide. Several targeting scan (3D T1-weighted) MR images were sequentially taken to aid in the positioning of the cannula guide in the planned anteroposterior and mediolateral planes. When the trajectory angle (anteroposterior mediolateral direction) of the fluid-filled cannula was confirmed to be on target, the alignment stem was locked into position and the system prepared for infusion. The fluid-filled cannula was removed and the remote introducer fastened to the stem. The guiding insert was placed in the guiding stem. The catheter for the infusion was threaded through the remote introducer and the guiding insert and fastened to the remote introducer by a locking mechanism.

A fused-silica single-endport cannula with a step and a silica stylet were used [3]. The dimensions were: tip outer diameter $=$ $0.35 \mathrm{~mm}$; tip inner diameter $=0.25 \mathrm{~mm}$; tip length $=3.0 \mathrm{~mm}$; shaft outer diameter $=0.65 \mathrm{~mm}$; shaft inner diameter $=0.32 \mathrm{~mm}$, and shaft length $=100.0 \mathrm{~mm}$. Silica infusion lines connected the catheter to $5-\mathrm{cm}^{3}$ syringes that were placed in an MRI-compatible syringe pump attached to the control mechanism of the standard Harvard Apparatus PHD 2000. Monitoring of the pressure in the infusion lines was performed using a pressure monitoring and infusion pump controller system from Engineering Resources Group Inc. The infusion lines were primed with degassed sterile phosphate-buffered saline (PBS) and then reversed filled with the viral vector suspension. After pressure of the lines was stabilized, the catheter was introduced into the brain under MRI monitoring, advancing the remote introducer at approximately $5-7 \mathrm{~mm} / \mathrm{min}$ until reaching the planned target. After the pressure was stabilized, the stylet was retracted $8 \mathrm{~mm}$. When the pressure stabilized again, the relative pressures were zeroed and the infusion started.

Two animals received low-titer infusions $\left(1 \times 10^{10} \mathrm{GC} / 30 \mu \mathrm{l}\right)$ of AAV5-GFP (left ventral postcommissural putamen) and AAV5GDNF (right ventral postcommissural putamen). The other 2 animals received high-titer infusions $\left(1 \times 10^{11} \mathrm{GC} / 30 \mu \mathrm{l}\right)$ of AAV5GFP (left putamen) and AAV5-GDNF (right putamen). The total volume for each inoculation was $30 \mu \mathrm{l}$ and it was administered at a flow rate of $1 \mu \mathrm{l} / \mathrm{min}$; $10 \mathrm{~min}$ after the first infusion was completed, the cannula was retracted and the targeting and infusion procedure for the second injection site begun. After the second infusion was completed and a $10-$ min postinfusion period had lapsed, the cannula was retracted and the animals were transported to the surgical room, where the bases were removed and the incision was closed in layers.

\section{Magnetic Resonance Imaging}

For MRI, the animals were food deprived overnight and initially sedated with ketamine $(10-15 \mathrm{mg} / \mathrm{kg}$, IM) followed by isoflurane $(1-3 \%)$ for the duration of the scan. Atropine (0.02-0.05 $\mathrm{mg} / \mathrm{kg}, \mathrm{IM}$ ) was also administered, as previously described [3]. Vital signs (heart rate and blood oxygen, respiration and temperature) were monitored during the procedure using MRI-compatible instruments. The animals were wrapped for warmth and placed in an MRI-compatible stereotactic frame. The placement of the monkeys' head in the frame was recorded.

Baseline and intraoperative imaging was performed in a 3-tesla GE Discovery MR750x device (GE Healthcare, Waukesha, Wis., USA). A custom 3-inch-diameter, receive-only surface coil (MR Instruments Inc., Minneapolis, Minn., USA) was used for scanning; 3D spoiled gradient echo images were acquired with TR/ $\mathrm{TE}=21 / 6 \mathrm{~ms}, \mathrm{NEX}=1$ and flip angles of $\alpha=[6,20]^{\circ}$. Images were acquired with alternating flip angles throughout the duration of the infusion, and then fitted to the spoiled gradient echo signal equation to yield quantitative maps of relaxation rate $(\mathrm{R} 1=1 / \mathrm{T} 1)$. $3 \mathrm{D}$ coronal volumes were obtained with a $0.625 \times 0.55$ resolution in-plane and 80 slices $0.8 \mathrm{~mm}$ thick. Masks of the infusion enhancement were manually drawn on the R1 maps slice by slice in order to map the apparent distribution and volume of the infusate. Optimal window/level values were selected on a case-by-case basis to enhance the contrast in the infusion area and to aid the drawing of the $3 \mathrm{D}$ volumetric masks. Final masks were used to outline the total infusion volume and distribution. The manual masking was performed blinded to the postmortem histological results.

\section{Necropsy and Preparation of Tissue}

Six weeks after the surgery, the monkeys were euthanized by transcardiac perfusion with heparinized PBS under pentobarbital anesthesia ( $\leq 35 \mathrm{mg} / \mathrm{kg}, \mathrm{IV})$. The brains were harvested and sliced in $4-\mathrm{mm}$ coronal slabs using a calibrated Plexiglas apparatus. Tissue punches ( $2 \mathrm{~mm}$ of the caudate, putamen and cortex) were obtained for biochemical analysis and placed in liquid nitrogen. The rest of the brain tissue was postfixed in $4 \%$ paraformaldehyde for $72 \mathrm{~h}$ and cryoprotected by immersion in a graded (10-30\%) sucrose/PBS solution. The tissue slabs were cut frozen $(40-\mu \mathrm{m}$ sections) on a sliding knife microtome. All sections were stored in a cryoprotectant solution before processing. Coronal brain sections were used for immunohistochemical staining according to our previously published protocols $[3,13]$. Antibodies used included GDNF (1:1,000; R\&D Systems, Minneapolis, Minn., USA), GFP (1:200; Millipore, Billerica, Mass., USA), neuron-specific nuclear protein (NeuN; 1:1,000; Millipore), glial fibrillary acidic protein (GFAP; 1:2,000; DakoCytomation, Glostrup, Denmark), tyrosine hydroxylase (TH; 1:20,000; ImmunoStar Inc., Hudson, Wis., USA) and CD68 (1:3,000; DakoCytomation). Nissl staining was also performed for the general evaluation of brain structures.

\section{Confocal Immunofluorescence}

Triple immunofluorescence experiments were performed to colocalize GFP with the neuronal marker NeuN, the astrocytic marker GFAP and/or the dopaminergic neuronal marker TH. Sections were first incubated in a blocking solution (5\% normal goat 
serum, $2 \%$ BSA and $0.3 \%$ Triton X-100 in TBS, $\mathrm{pH} 7.4$ ) for $1 \mathrm{~h}$ to inhibit background staining, followed by primary chicken polyclonal anti-GFP (1:500; DakoCytomation), primary mouse monoclonal anti-NeuN (1:1,000; Millipore) and primary rabbit polyclonal anti-GFAP (1:2,000; Millipore) for $24 \mathrm{~h}$ at $4^{\circ} \mathrm{C}$. After 3 washes, sections were incubated in alexafluor 488 conjugated secondary goat anti-chicken IgG for GFP (1:200; Millipore), alexafluor 594 conjugated secondary goat anti-mouse IgG for NeuN (1:200; Millipore), and alexafluor 647 conjugated secondary goat anti-rabbit IgG for GFAP (1:200; DakoCytomation) for $1 \mathrm{~h}$. Following the reactions, the slides containing the tissue were coverslipped. Confocal images were obtained for each animal at $\times 20$ and $\times 60$ magnification using a Nikon E1 confocal microscope (NISElements software).

\section{Evaluation of Immunohistochemistry}

Neuroanatomical mapping of GFP and GDNF expression was performed in 6 matched and equally spaced coronal brain sections by an investigator blinded to the vector titer. The evaluated areas included the putamen, dorsal prefrontal cortex globus pallidus, claustrum, subthalamic nucleus, SN, thalamus and entorhinal cortex. The nuclei were identified using a Nikon Eclipse E800 microscope and a stereotactic atlas as a reference [14]. Using a modified rating scale [15], protein expression was classified as: - 'not detected', \pm 'minimal' (only few positive cells or fibers are found), + 'moderate' ( $<25 \%$ of the nucleus is covered by strong immunoreactivity), ++ 'intense' $(<50 \%$ of the nucleus is covered by strong immunoreactivity) and +++ 'very intense' ( $>50 \%$ of the nucleus is covered by strong immunoreactivity).

The optical density (OD) and area of GDNF, GFP and TH immunoreactivity were quantified using NIH ImageJ software, as previously described [16]. Images of coronal sections were captured for each monkey, using an Epson Expression 1640XL-GA high-resolution digital scanner. ImageJ was calibrated using a step tablet, gray scale values were converted to OD units using the Rodbard function, and the mean OD for each area of interest was recorded. OD was evaluated with a threshold in pixels of 0.15 for GDNF, 0.15 for GFP and 0.07 for striatal TH defining the areas of immunoreactivity.

The total number of $\mathrm{TH}$-immunoreactive (TH-ir) neurons in the right and left SN was calculated using unbiased stereological cell-counting methods previously described. The optical dissector system consisted of a computer-assisted image analysis system including a Zeiss Axioplan 2 imaging photomicroscope (Carl Zeiss Inc.) hard coupled to a MAC5000 high-precision computer-controlled $\mathrm{x}-\mathrm{y}-\mathrm{z}$ motorized stage and a MicroFire CX9000 camera (Optronics, Goleta, Calif., USA). Neuronal counts were performed using Stereo Investigator version 7.5 (MicroBrightField, Williston, Vt., USA). The SN was outlined under low magnification $(\times 2.5)$. The total number of $\mathrm{TH}$-ir neurons within the counting frame was counted using a $\times 100$ oil immersion objective with a 1.4 numerical aperture. Six equally spaced sections from each subject containing the $\mathrm{SN}$ were used for analysis.

\section{GDNF ELISA and Brain Tissue qPCR Analysis}

Frozen tissue punches from the caudate, putamen and cortex were powdered in a frozen state in a CP-02 crushing device (Covaris, Woburn, Mass., USA) and divided into 2 portions. One portion was used for isolation of GDNF protein and the other one for DNA isolation. For protein isolation, the powder was lysed in lysis buffer containing $0.1 \%$ Tween $20,0.5 \%$ BSA and 2 mM EDTA supplemented with complete protease inhibitor tablets (Roche Diagnostics Corp., Indianapolis, Ind., USA) in PBS. The GDNF concentration (expressed as nanograms of GDNF per milligram protein) was determined by GDNF ELISA (R\&D Systems) according to the manufacturer's protocol $[13,16]$. A low-range advanced Lowry Protein Assay Kit (Bio-Rad Laboratories, Hercules, Calif., USA) was used to determine the amount of total protein in the sample.

To determine the amount of vector GC present in the samples, genomic (g) DNA was isolated using a Gentra Puregene kit for tissues (Qiagen, Valencia, Calif., USA). Glycogen was added according to the manufacturer's instructions in order to aid gDNA precipitation. The primers used for detection of the GDNF sequence were $\left(5^{\prime}-3^{\prime}\right)$ : GDNF forward - GCGCTGAGCAGTGACTCAAA; GDNF reverse - CCATGACATCATCGAACTGATCA, and GDNF probe - TGCCAGAGGATTATC. The GDNF probe was labeled $5^{\prime}$ with 6-carboxyfluorescein (6-FAM) and $3^{\prime}$ with a minor groove binder/nonfluorescent quencher tandem (Applied Biosystems, Carlsbad, Calif., USA). The primers used for detection of the enhanced GFP (EGFP) sequence were: EGFP forward - AGCAAAGACCCCAACGAGAA; EGFP reverse - GCGGCGGTCACGAACTC, and EGFP probe - CGCGATCACATGGTCCTGCT. The EGFP probe was labeled $5^{\prime}$ with 6-FAM and $3^{\prime}$ with tetramethylrhodamine. The primers used for the detection of the endogenous monkey porphobilinogen deaminase (PBGD) sequence were: PBGD forward - GATGCACGGCTCTAGATTTAGTGA; PBGD reverse - AATGAAAGGACCACGTCTGTGTAG, and PBGD probe - ACCGCGAACGTTC. The PBGD probe was labeled $5^{\prime}$ with 6 -FAM and $3^{\prime}$ with a minor groove binder/ nonfluorescent quencher tandem.

qPCR was performed on $250 \mu \mathrm{g}$ of gDNA using an Applied Biosystems TaqMan Fast Universal PCR master mix and the relevant primers and probes at an end concentration of 300 and 200 $\mathrm{nM}$, respectively, in a total reaction volume of $20 \mu \mathrm{l}$. Reactions were run on a 7500 Fast cycler (Applied Biosystems) as follows: $20 \mathrm{~s}$ at $95^{\circ} \mathrm{C}$, followed by 40 cycles of $3 \mathrm{~s}$ at $95^{\circ} \mathrm{C}$ and $30 \mathrm{~s}$ at $60^{\circ} \mathrm{C}$. The PBGD qPCR results were used to confirm the absence of PCR inhibitors in the samples and to ensure proper gDNA loading; in the absence of inhibitors and at a constant amount of input gDNA, the PBGD Cq values should be tightly clustered. For target sequence quantification, a standard curve was included in each analysis, which consisted of a dilution series of a plasmid containing the relevant target sequence (i.e. GDNF or GFP). Absolute target sequence copy numbers in the samples were calculated from their $\mathrm{Cq}$ values via interpolation from the relevant plasmid standard curve, and were expressed as GC per microgram gDNA.

\section{Results}

Neuronal Gene Expression Was Found in the Areas of CED Infusion

IMRI confirmed a targeting position in the ventral postcommissural putamen. MRI monitoring during infusions recorded a limited pattern of distribution that irradiated from the catheter tip, filling the postcommissur- 


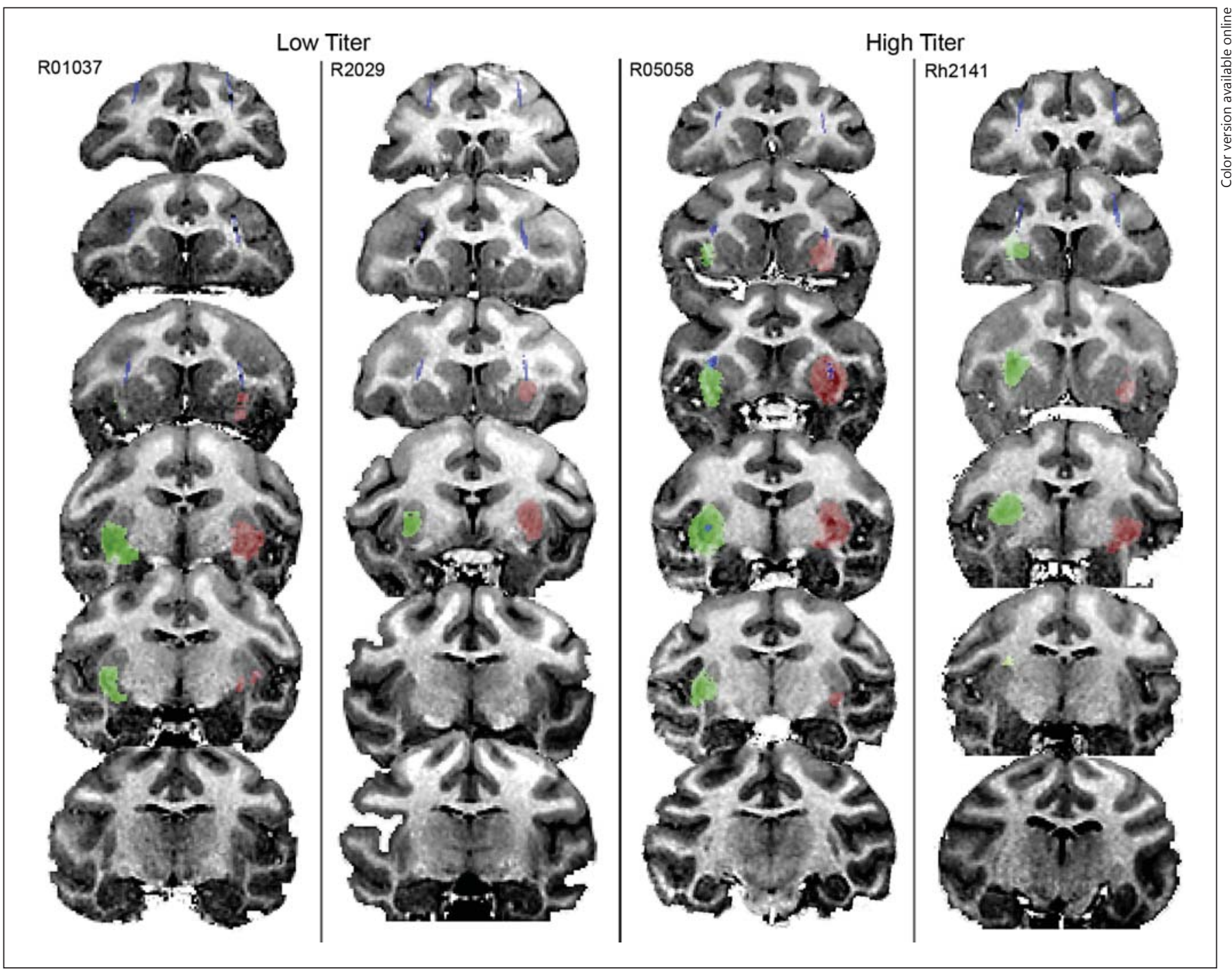

Fig. 1. Viral vector infusions can be identified by MRI without contrast agent. Sequential T1-weighted coronal brain MR images of 4 rhesus monkeys obtained after completion of CED of a 30- $\mu$ suspension of AAV5-GFP (left hemisphere; green; colors in online version only) and GDNF (right hemisphere; red) into the ventral postcommissural putamen nucleus. Some backflow (fine line protruding; blue) is observed.

al putamen with some spilling into the surrounding lateral white matter (fig. 1).

Six weeks after surgery, the animals were euthanized and their brains processed. Colocalization of GFP and GFAP (astrocytic marker) or NeuN (neuronal marker) immunofluorescence in the putamen nucleus confirmed gene expression and showed that the majority of the transfected cells were neurons (fig. 2). CD68 (microglia/ macrophage marker) and GFAP immunoreactivity (astroglia marker; both measures of host immunoreaction) was minimal and limited to the needle tracts and adjacent white matter, including the external capsule and corpus callosum (not shown).

Coronal brain sections immunostained for GFP (fig. 3) or GDNF (fig. 4) showed positive protein expression, each limited to the corresponding hemisphere injected. The most intense gene expression was observed at the injection site in the ventral postcommissural putamen, and from there, the expression spread to nearby regions such as the external capsule, claustrum, internal laminae and most of the lateral pallidum boundary. Staining was also found following the needle tract in the 


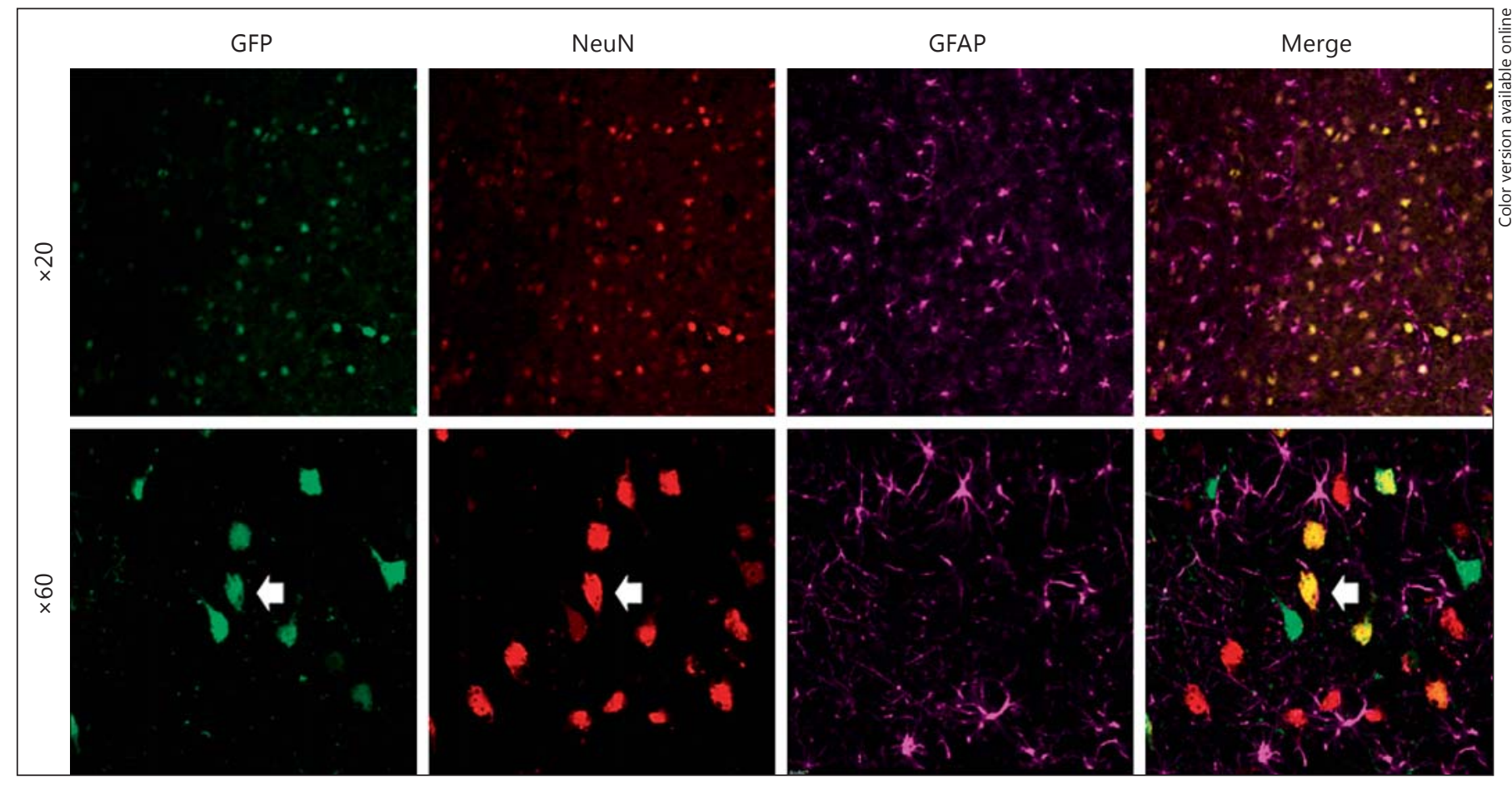

Fig. 2. GFP was expressed mostly in neurons. High- and low-titer fluorescent images of the putamen nucleus injected with AAV5-GFP (green) immunostained for NeuN (red) and GFAP (purple). White arrows: example of a positive neuron expressing GFP (yellow).

Table 1. Neuroanatomical distribution and intensity of gene expression after CED of AAV5 encoding for GFP or GDNF into the ventral postcommissural putamen nucleus

\begin{tabular}{|c|c|c|c|c|c|c|c|c|}
\hline & \multicolumn{4}{|c|}{ Low titer } & \multicolumn{4}{|c|}{ High titer } \\
\hline & \multicolumn{2}{|c|}{ R02029 } & \multicolumn{2}{|c|}{ R01037 } & \multicolumn{2}{|c|}{ R05058 } & \multicolumn{2}{|c|}{ Rh2141 } \\
\hline & GFP & GDNF & GFP & GDNF & GFP & GDNF & GFP & GDNF \\
\hline Putamen & ++ & + & + & ++ & ++ & +++ & ++ & +++ \\
\hline Dorsal prefrontal cortex & \pm & \pm & \pm & + & ++ & ++ & + & ++ \\
\hline Globus pallidus & - & - & \pm & \pm & + & + & + & + \\
\hline Claustrum & \pm & - & \pm & - & + & + & + & + \\
\hline Subthalamic nucleus & - & - & - & - & - & \pm & - & \pm \\
\hline $\mathrm{SN}$ & \pm & \pm & \pm & \pm & ++ & ++ & + & + \\
\hline Thalamus & - & - & - & - & \pm & \pm & - & - \\
\hline Entorhinal cortex & - & - & - & - & \pm & \pm & - & \pm \\
\hline
\end{tabular}

Gene expression was scored as: - 'not detected', \pm 'minimal', + 'moderate', ++ 'intense' and +++ 'very intense'.

corpus callosum, subcortical white matter and frontal cortex (table 1). The distribution of gene expression also followed the shape of the putaminal lenticulostriate artery into ventral white matter structures. Greater GFP-ir and GDNF-ir expression volumes and OD were found in the high-titer than in the low-titer monkeys (table 2).

GDNF ELISA and qPCR analysis were performed on tissue punches of the ventral and dorsal putamen, caudate, globus pallidus and cortex obtained from coronal 


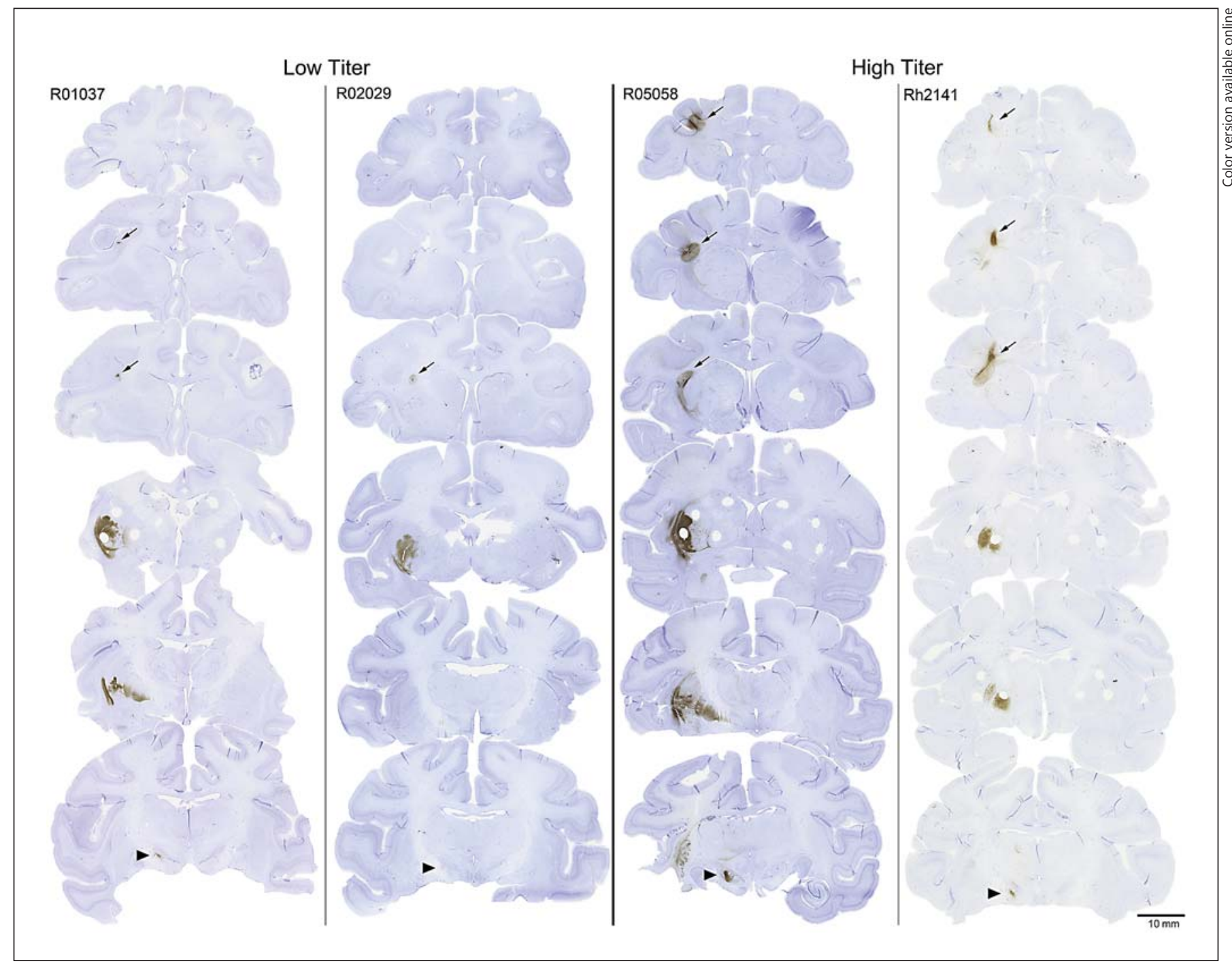

Fig. 3. Distribution of GFP expression followed the infusion pattern and neural network. Sequential images of GFP-immunostained coronal brain sections of 4 rhesus monkeys that received an inoculation of AAV5-GFP into the left ventral postcommissural putamen nucleus, and one of AAV5-GDNF in the right, using CED methods. Positive immunostaining in needle tracks (arrows) or SN (arrowheads).

Table 2. Quantification of putaminal GFP and GDNF gene expression as well as local TH response

\begin{tabular}{|c|c|c|c|c|c|c|c|c|}
\hline & \multicolumn{4}{|l|}{ Low titer } & \multicolumn{4}{|l|}{ High titer } \\
\hline & \multicolumn{2}{|l|}{ R02029 } & \multicolumn{2}{|l|}{ R01037 } & \multicolumn{2}{|l|}{ R05058 } & \multicolumn{2}{|l|}{ Rh2141 } \\
\hline & GFP & GDNF & GFP & GDNF & GFP & GDNF & GFP & GDNF \\
\hline $\begin{array}{l}\text { Percent covered by expressed } \\
\text { gene }\end{array}$ & 29.51 & 14.21 & 11.81 & 19.95 & 31.87 & 41.2 & 29.59 & 20.22 \\
\hline Gene expression, OD & $0.016 \pm 0.011$ & $0.226 \pm 0.009$ & $0.339 \pm 0.015$ & $0.314 \pm 0.016$ & $0.454 \pm 0.019$ & $0.458 \pm 0.012$ & $0.413 \pm 0.008$ & $0.355 \pm 0.017$ \\
\hline $\mathrm{TH}, \mathrm{OD}$ & $0.079 \pm 0.001$ & $0.062 \pm 0.007$ & $0.082 \pm 0.004$ & $0.094 \pm 0.006$ & $0.144 \pm 0.006$ & $0.226 \pm 0.009$ & $0.116 \pm 0.006$ & $0.176 \pm 0.006$ \\
\hline
\end{tabular}

The 4 rhesus monkeys received an inoculation of AAV5-GFP (left) and AAV5-GDNF (right) in each ventral postcommissural putamen nucleus, using the CED method. 


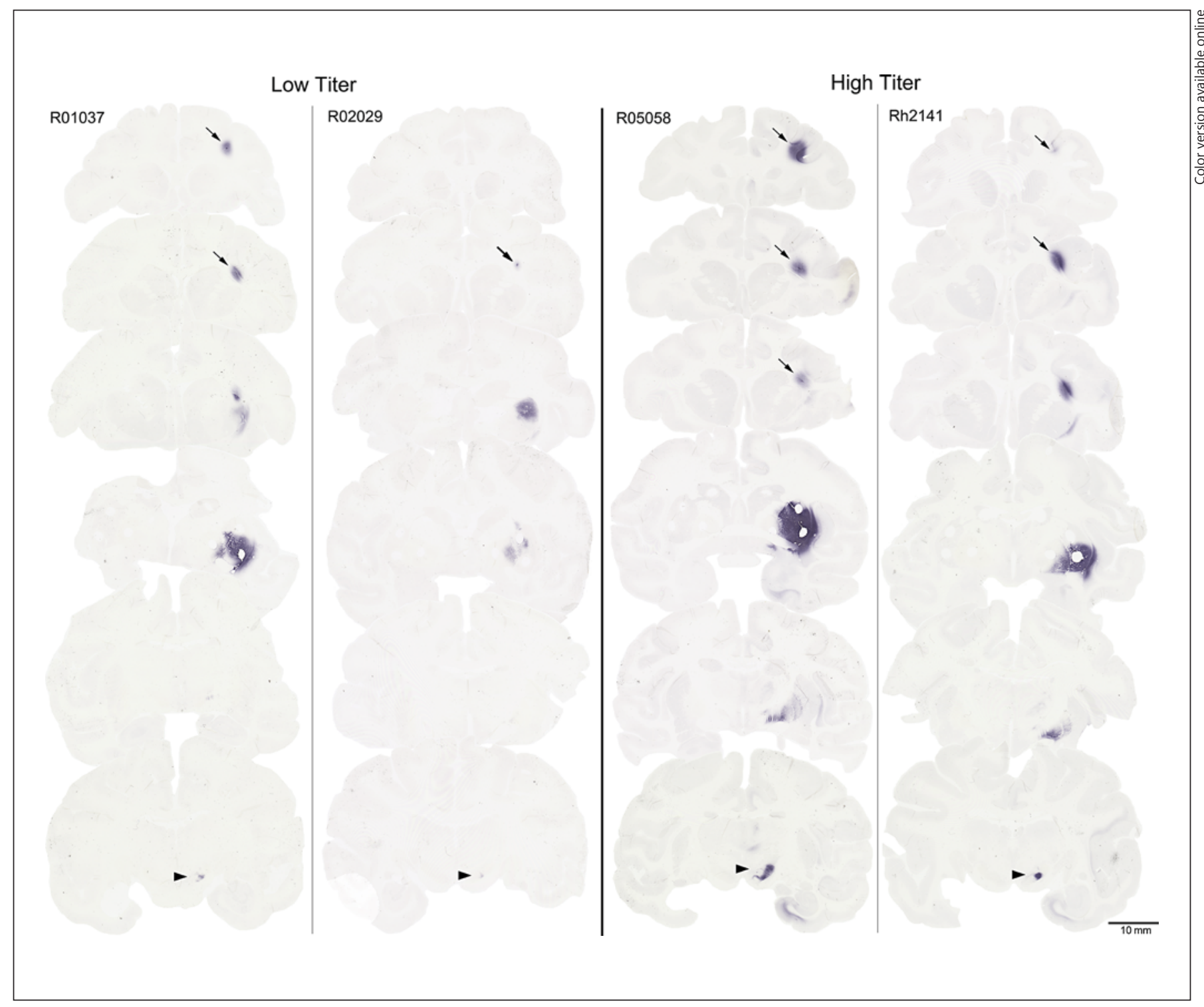

Fig. 4. Distribution of GDNF expression followed the infusion pattern and neural network. Sequential images of GDNF-immunostained coronal brain sections of 4 rhesus monkeys that received an inoculation of AAV5-GFP in the left ventral postcommissural putamen nucleus, and one of AAV5-GDNF in the right, using CED methods. Positive immunostaining in needle tracks (arrows) or SN (arrowheads).

sections at the level of the postcommissural putamen. GDNF protein expression was maximal at the injection site in all animals, followed by the dorsal putamen. Low levels of expression were also found in the globus pallidus and, in the case of one animal (R05058), in the caudate nucleus. The concentrations of GDNF were dependent on the titer that was injected. GDNF qPCR showed a significant positive correlation (Pearson's correlation: $\mathrm{R}^{2}=$ 0.8670 ; $p<0.0001$; fig. 5) between the number of AAV5-
GDNF GC and the protein concentration obtained by ELISA.

\section{GFP and GDNF Expression Was Also Observed in the} Putaminal Neuronal Network

In addition to gene expression in the areas which the infusion cloud reached, GFP and GDNF immunostaining was also found in the globus pallidus, thalamus, nucleus accumbens, subthalamic nucleus and SN (fig. 2, 3; ta- 
Table 3. Stereological quantification of GFP-, GDNF- and TH-positive neurons in the SN of 4 rhesus monkeys that had AAV5-GFP or GDNF delivered into the ventral postcommissural putamen nucleus

\begin{tabular}{|c|c|c|c|c|c|c|c|c|}
\hline \multirow[b]{3}{*}{ Immunostaining } & \multicolumn{4}{|l|}{ Low titer } & \multicolumn{4}{|c|}{ High titer } \\
\hline & \multicolumn{2}{|l|}{ R02029 } & \multicolumn{2}{|l|}{ R01037 } & \multicolumn{2}{|l|}{ R05058 } & \multicolumn{2}{|l|}{ Rh2141 } \\
\hline & GFP & GDNF & GFP & GDNF & GFP & GDNF & GFP & GDNF \\
\hline GFP, cell number & - & - & 618 & - & 1,226 & - & 2,450 & - \\
\hline GDNF, cell number & - & 2,599 & - & 4,339 & - & 27,788 & - & 4,773 \\
\hline TH, cell number & 137,987 & 132,971 & 149,773 & 146,096 & 124,766 & 140,760 & 139,685 & 139,786 \\
\hline $\mathrm{TH}$, cell volume & 9,660 & 11,559 & 11,856 & 14,722 & 10,466 & 14,407 & 9,334 & 12,627 \\
\hline
\end{tabular}

Numbers are nigral cell counts.

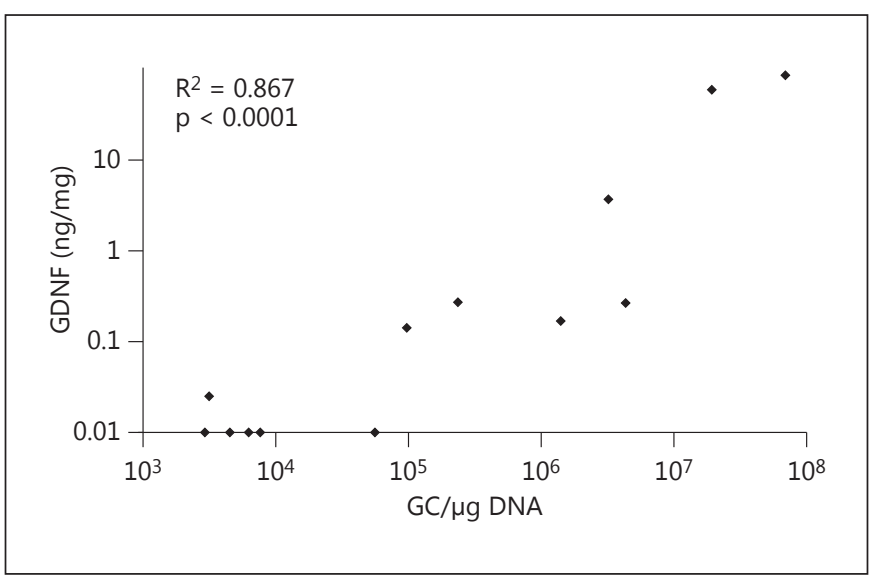

Fig. 5. Pearson's correlation graph of GDNF qPCR and ELISA data. The number of GDNF GC correlated with the amount of GDNF protein.

ble 1). This expression was not observed on the MR images. Closer analysis of the $\mathrm{SN}$ showed GFP staining to be limited to positive fibers in the $\mathrm{SN}$ pars reticulata (SNpr) and a few GFP-positive neurons in the SNpc (fig. 6; table 3). GDNF expression was also abundant in SNpr fibers, but it could also be seen in the neuropil, in many $\mathrm{SNpc}$ and SNpr neurons and in astrocytes (fig. 6; table 3).

\section{GDNF but Not GFP Expression Was Associated with Increased Nigrostriatal TH-ir}

As GDNF exerts trophic effects on dopaminergic neurons, we evaluated whether AAV5-GDNF affected nigrostriatal TH expression. Qualitatively, an asymmetric expression of $\mathrm{TH}$ in the striatum and $\mathrm{SN}$ was observed in all four animals but was more evident in the high-titer monkeys (fig. 7). Quantification of TH OD confirmed that the right putamen had more staining than the left (table 2) and that the differences were associated with GDNF expression and vector titer. Stereological nigral cell counts of TH-ir neurons did not show differences between treatments, although the cell volume was greater in the AAV5-GDNF-treated side than in the AAV5-GFPtreated side (table 3 ).

\section{Discussion}

Our results demonstrate that CED combined with IMRI targeting is a replicable method for accurately delivering viral vectors into the nonhuman primate brain. Our data also show that the final pattern of gene expression is defined by infusate distribution, viral vector titer, the nature of the gene product and the neuroanatomy of the area to which the gene is delivered. Note that the infusions in this study were performed without the use of a T1-shortening contrast agent with gadolinium as was done in other studies. While the sensitivity of the infusion is much lower without the contrast agent, this study demonstrated that it is possible to reliably detect and map the distribution of an infusion without contrast agents. This is important as the coinfusion of contrast agents with viral vectors may influence the distribution and expression of the latter [17].

The finding that the viral vector titer affects the pattern of distribution of the gene product is novel. It suggests an interaction between vector and host during infusion that affects which cells are transfected. Several studies (mostly on rodents) had assessed a dose-dependent effect of gene therapy [18], yet an impact of titer on distribution has not been described. Gene therapy aiming to deliver therapeutic molecules to large structures such as 
Fig. 6. GFP and GDNF were differentially expressed in the $\mathrm{SN}$. Images of the $\mathrm{SN}$ at the level of the emergence of the 3rd nerve, immunostained for GFP (A-C) or GDNF (DF) and counterstained with Nissl, of a monkey that received an inoculation of AAV5GFP in the left ventral postcommissural putamen nucleus, and one of AAV5-GDNF in the right, using CED methods. Top and bottom white squares in $\mathbf{A}$ and $\mathbf{D}$ correspond to the high-magnification images in B, C and E, F, respectively. B, C Arrows: GFP-negative cell bodies. E, F Arrows: GDNF-positive cell bodies. $\mathbf{D}$ Scale bar = $500 \mu \mathrm{m}$. F Scale bar $=50 \mu \mathrm{m}$.

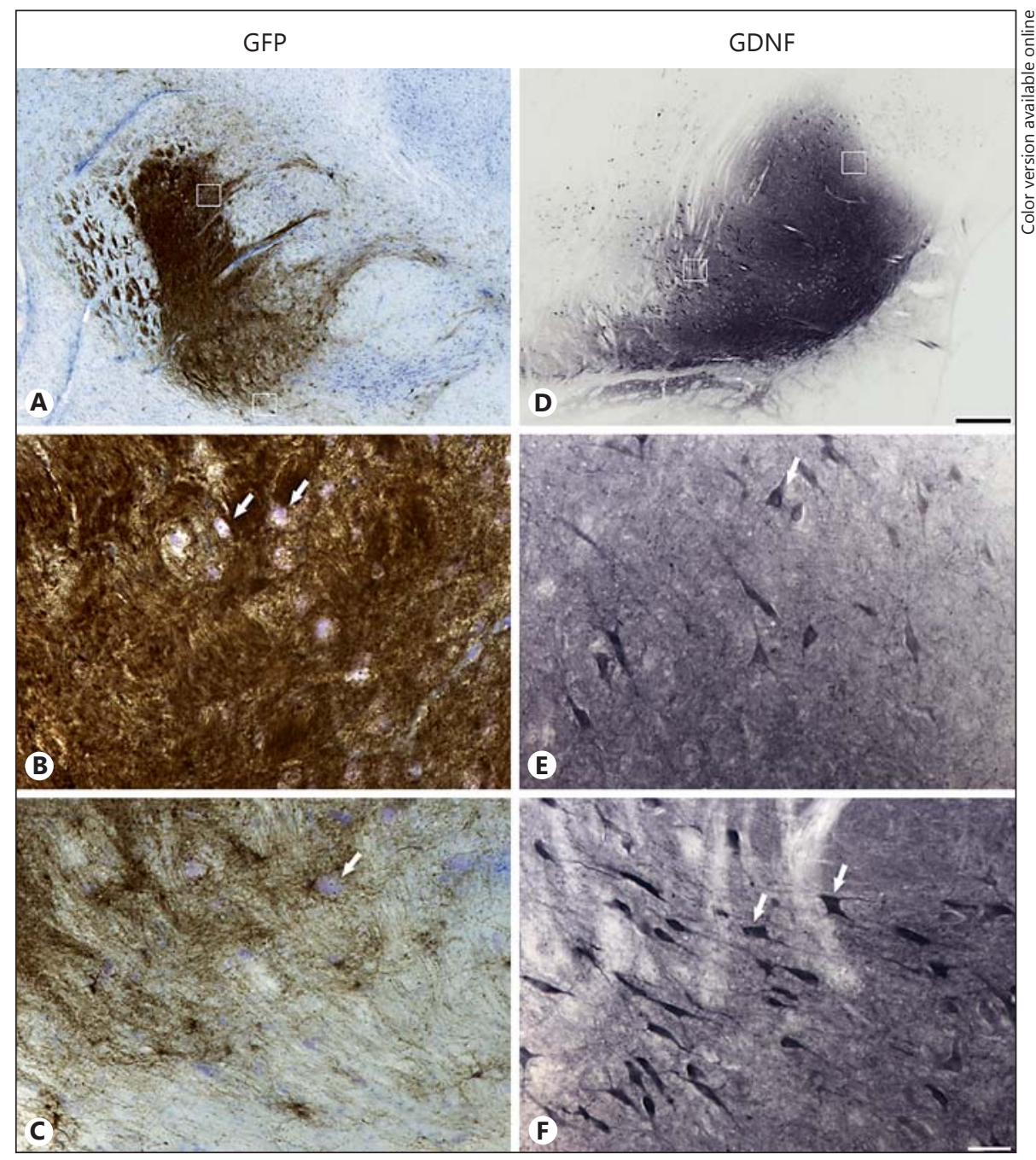

the putamen nucleus has been affected by limited dispersion [10]. Different methods, including pressure-driven infusion by CED, have been proposed to increase the area of viral vector distribution by a single inoculation [19]. Mannitol coinfused with AAV2 has been successfully used in preclinical $[20,21]$ and clinical trials [22] to increase vector distribution. A possible explanation for this effect could be that hyperosmolar solutions such as mannitol increase the extracellular space volume fraction while decreasing tortuosity (a measure of diffusional hindrance), facilitating the distribution of the viral vector [23]. Coinfusion of heparin with AAV2 also increases viral vector spread [24]. AAV2 and heparin compete for binding to heparan sulfate proteoglycan cellular receptors; therefore, their coinfusion increases AAV2 distribution. Although heparin use has been limited due to an increased risk of bleeding, heparin studies highlight the impact of viral vector binding to specific receptors on their distribution [25].

In our study, we found that delivery of the same volume of a high titer, compared with a low titer, of AAV5 viral vector suspension increases the distribution pattern of protein expression. This suggests that a phenomenon similar to competition for receptor occupancy occurred. Although a specific AAV5 receptor has not yet been identified, it is known that the presence of sialic acid residues in cellular membranes increases AAV5 cellular transfection [26]. The infusion of a higher viral vector titer magnified misallocation of the infusate. We chose to inject a final volume of $30 \mu \mathrm{l}$ at $1 \mu \mathrm{l} / \mathrm{min}$ into the ventral postcommissural putamen based on our work with gadolinium solution $[3,4]$. In our previous studies, a volume of $>30$ 


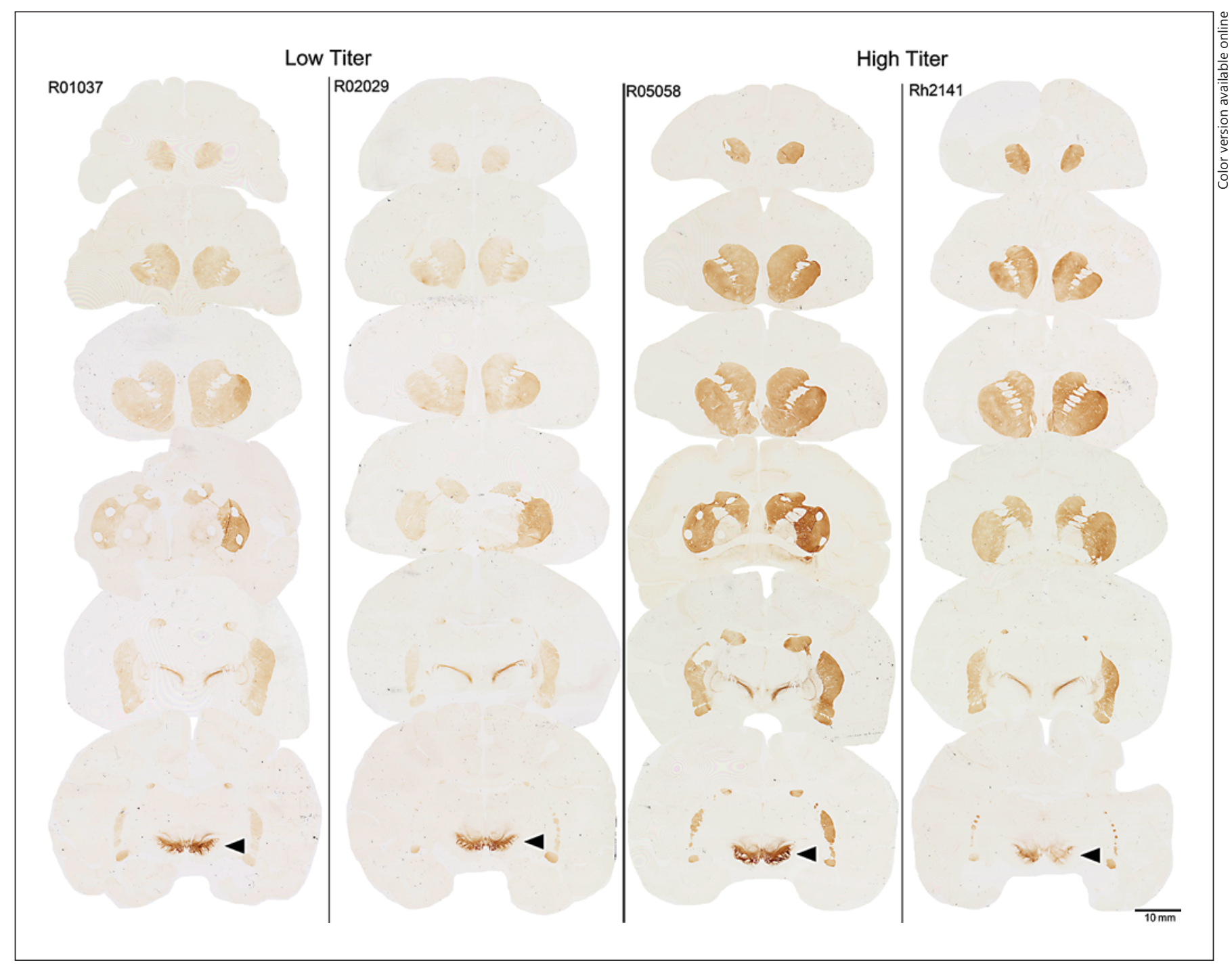

Fig. 7. GDNF expression enhanced normal TH immunoreactivity. Sequential images of TH-immunostained coronal brain sections of 4 rhesus monkeys that received an inoculation of AAV5-GFP in the left ventral postcommissural putamen nucleus, and one of AAV5-GDNF in the right, using CED methods. Arrowheads: SN with increased TH immunostaining.

$\mu \mathrm{l}$ spills over the natural boundaries of the ventral putamen nucleus. While all of our animals received precisely $30 \mu \mathrm{l}$ at a similar location, gene expression was observed beyond the external capsule and internal laminae with a higher titer, especially with GDNF. In addition, backflow following the needle track is commonly observed during intracerebral infusions. By improving the infusion parameters and technology, we have been able to minimize this backflow, yet we could not completely eliminate it. Although the backflow observed by MRI was minimal and similar in all cases, the animals that received the high- er titer showed increased GDNF expression in the needle track compared with the animals receiving the lower titers. These findings suggest that a more replicable infusion pattern can be obtained with a lower titer and that increased gene expression should be evaluated against the risk of side effects.

Expression of GFP and GDNF occurred in brain nuclei that received or sent putaminal projections. Secondary distribution was not detected during IMRI. AAV5 neurotropism $[27,28]$ indicates that after neuronal uptake, the vector and/or its encoded product can be antero- 
gradely or retrogradely transported from the original target to other brain regions. Neural network transport has several implications for planning and evaluating gene therapy strategies. First, neural network spreading can be advantageous for therapeutic distribution, yet reaching secondary targets may have unwanted effects. Second, if localized therapies are needed, vectors with glial tropism or ex vivo gene therapy approaches may be more appropriate. Third, the state of the host neural network may affect intra- and transneuronal transport. The current study was performed on normal animals whose neural networks were intact; yet patients with neurodegenerative disorders may have a compromised neural network distribution. While IMRI cannot detect secondary distribution, MRI plus diffusion tensor imaging can be used to evaluate neuronal tracts and estimate the integrity of the network. New studies on animal models to evaluate the effect of disease on the neural network distribution of vectors and proteins after gene therapy are warranted.

Because of our interest in PD, in this study, we surgically targeted the putamen nucleus and focused our analysis on the putaminal-nigral network [29]. A striking finding was the different pattern of expression of GFP and GDNF in the SN after putaminal delivery. GFP expression was strongly present in the axons of neurons projecting from the putamen into the SNpr. Only a few GFPpositive neuronal cell bodies could be identified in the $\mathrm{SNpc}$, and their number was greater in the high-titer animals. Fiber expression confirmed previous descriptions of GFP as a robust anterograde transport marker [6], as it is not secreted. The minimal number of GFP-positive nigral cell bodies suggests that after intraputaminal infusion, the AAV5 vectors which did not transfect local putaminal neurons were taken up by the terminals of nigral neurons and then retrogradely transported to the cell bodies. This would imply that the higher titer increased the localized availability of the vector, facilitated uptake and ultimately increased nigral cell expression. In comparison, GDNF expression was found to be abundant inside nigral neurons. Based on the AAV5-GFP data, we propose that a minimal amount of vector encoding for GDNF was retrogradely transported and that GDNF protein, which was expressed and released by putaminal terminals ending at the nigra, was taken up by local neurons.

\section{Conclusion}

Our results demonstrate that after controlling for target and infusate volume, the intracerebral distribution of a gene product is affected by the vector titer and product biology. These findings are particularly valuable for clinical application, where the safety and efficacy of a treatment is defined by accurate delivery of a gene product.

\section{Acknowledgements}

This research was supported by the Jeffrey L. Morse living trust, the Kinetics Foundation and NIH-NCRR grant P51 RR000167 (Wisconsin National Primate Research Center, University of Wisconsin). This research was conducted at a facility constructed with support from Research Facilities Improvement Program grants RR15459-01 and RR020141-01. We thank Joel A. Shires for assistance during morphological processing and analysis.

\section{Disclosure Statement}

uniQure (formerly AMT) provided the viral vectors used in this study. Marc Sonnemans, Stephan Hermening and Bas Blits were employees of AMT during the experiment.

Andrew Alexander is part owner of inseRT, Inc., which did not contribute to this study.

\section{References}

-1 Sampson JH, Raghavan R, Brady M, Friedman AH, Bigner D: Convection-enhanced delivery. J Neurosurg 2011;115:463-464, discussion 465-466.

2 Kimmelman J, Duckworth K, Ramsay T, Voss $\mathrm{T}$, Ravina B, et al: Risk of surgical delivery to deep nuclei: a meta-analysis. Mov Disord 2011;26:1415-1421.

-3 Emborg ME, Joers V, Fisher R, Brunner K, Carter V, et al: Intraoperative intracerebral MRI-guided navigation for accurate targeting in nonhuman primates. Cell Transplant 2010; 19:1587-1597.

Distribution of Gene Expression after CED
4 Brady ML, Raghavan R, Alexander A, Kubota $\mathrm{K}$, Sillay K, et al: Pathways of infusate loss during convection-enhanced delivery into the putamen nucleus. Stereotact Funct Neurosurg 2013;91:69-78.

5 San Sebastian W, Richardson RM, Kells AP, Lamarre C, Bringas J, et al: Safety and tolerability of magnetic resonance imaging-guided convection-enhanced delivery of AAV2hAADC with a novel delivery platform in nonhuman primate striatum. Hum Gene Ther 2012;23:210-217.
6 Burger C, Gorbatyuk OS, Velardo MJ, Peden CS, Williams P, et al: Recombinant AAV viral vectors pseudotyped with viral capsids from serotypes 1,2 , and 5 display differential efficiency and cell tropism after delivery to different regions of the central nervous system. $\mathrm{Mol}$ Ther 2004;10:302-317.

7 Peden CS, Burger C, Muzyczka N, Mandel RJ: Circulating anti-wild-type adeno-associated virus type 2 (AAV2) antibodies inhibit recombinant AAV2 (rAAV2)-mediated, but not rAAV5-mediated, gene transfer in the brain. J Virol 2004;78:6344-6359. 
8 Su X, Kells AP, Salegio EA, Richardson RM, Hadaczek P, et al: Real-time MR imaging with gadoteridol predicts distribution of transgenes after convection-enhanced delivery of AAV2 vectors. Mol Ther 2010;18:1490-1495.

-9 Morrison PF, Lonser RR, Oldfield EH: Convective delivery of glial cell line-derived neurotrophic factor in the human putamen. J Neurosurg 2007;107:74-83.

10 Marks WJ Jr, Bartus RT, Siffert J, Davis CS, Lozano A, et al: Gene delivery of AAV2-neurturin for Parkinson's disease: a double-blind, randomised, controlled trial. Lancet Neurol 2010;9:1164-1172.

11 Urabe M, Nakakura T, Xin KQ, Obara Y, Mizukami H, et al: Scalable generation of high-titer recombinant adeno-associated virus type 5 in insect cells. J Virol 2006;80:18741885.

-12 Unzu C, Hervas-Stubbs S, Sampedro A, Mauleon I, Mancheno U, et al: Transient and intensive pharmacological immunosuppression fails to improve AAV-based liver gene transfer in non-human primates. J Transl Med 2012;10:122.

13 Emborg ME, Moirano J, Raschke J, Bondarenko V, Zufferey R, et al: Response of aged parkinsonian monkeys to in vivo gene transfer of GDNF. Neurobiol Dis 2009;36:303311.

14 Paxinos G: The Rhesus Monkey Brain in Stereotaxic Coordinates. Waltham, Academic Press, 2000

15 Swanson CR, Emborg ME: Expression of peroxisome proliferator-activated receptor- $\gamma$ in the substantia nigra of hemiparkinsonian nonhuman primates. Neurol Res 2013, Epub ahead of print.
6 Ohshima-Hosoyama S, Simmons HA, Goecks $\mathrm{N}$, Joers V, Swanson CR, et al: A monoclonal antibody-GDNF fusion protein is not neuroprotective and is associated with proliferative pancreatic lesions in parkinsonian monkeys. PLoS One 2012;7:e39036.

17 Hullinger R, Ugalde J, Puron-Sierra L, Osting S, Burger C: The MRI contrast agent gadoteridol enhances distribution of rAAV1 in the rat hippocampus. Gene Ther 2013;20:11711177.

18 Gasmi M, Herzog CD, Brandon EP, Cunningham JJ, Ramirez GA, et al: Striatal delivery of neurturin by CERE-120, an AAV2 vector for the treatment of dopaminergic neuron degeneration in Parkinson's disease. Mol Ther 2007;15:62-68.

19 Carty N, Lee D, Dickey C, Ceballos-Diaz C, Jansen-West $\mathrm{K}$, et al: Convection-enhanced delivery and systemic mannitol increase gene product distribution of AAV vectors 5, 8, and 9 and increase gene product in the adult mouse brain. J Neurosci Methods 2010;194: 144-153.

20 Burger C, Nguyen FN, Deng J, Mandel RJ: Systemic mannitol-induced hyperosmolality amplifies rAAV2-mediated striatal transduction to a greater extent than local co-infusion. Mol Ther 2005;11:327-331.

21 Emborg ME, Carbon M, Holden JE, During MJ, Ma Y, et al: Subthalamic glutamic acid decarboxylase gene therapy: changes in motor function and cortical metabolism. J Cereb Blood Flow Metab 2007;27:501-509.

22 LeWitt PA, Rezai AR, Leehey MA, Ojemann SG, Flaherty AW, et al: AAV2-GAD gene therapy for advanced Parkinson's disease: a double-blind, sham-surgery controlled, randomised trial. Lancet Neurol 2011;10:309319.
23 Wolak DJ, Thorne RG: Diffusion of macromolecules in the brain: implications for drug delivery. Mol Pharm 2013;10:1492-1504.

24 Hamilton JF, Morrison PF, Chen MY, Harvey-White J, Pernaute RS, et al: Heparin coinfusion during convection-enhanced delivery (CED) increases the distribution of the glialderived neurotrophic factor (GDNF) ligand family in rat striatum and enhances the pharmacological activity of neurturin. Exp Neurol 2001;168:155-161.

25 Opie SR, Warrington KH Jr, Agbandje-McKenna M, Zolotukhin S, Muzyczka N: Identification of amino acid residues in the capsid proteins of adeno-associated virus type 2 that contribute to heparan sulfate proteoglycan binding. J Virol 2003;77:6995-7006.

26 Seiler MP, Miller AD, Zabner J, Halbert CL: Adeno-associated virus types 5 and 6 use distinct receptors for cell entry. Hum Gene Ther 2006;17:10-19.

27 Dodiya HB, Bjorklund T, Stansell J 3rd, Mandel RJ, Kirik D, et al: Differential transduction following basal ganglia administration of distinct pseudotyped AAV capsid serotypes in nonhuman primates. Mol Ther 2010;18:579587.

28 Markakis EA, Vives KP, Bober J, Leichtle S, Leranth C, et al: Comparative transduction efficiency of AAV vector serotypes 1-6 in the substantia nigra and striatum of the primate brain. Mol Ther 2010;18:588-593.

29 Hedreen JC, DeLong MR: Organization of striatopallidal, striatonigral, and nigrostriatal projections in the macaque. J Comp Neurol 1991;304:569-595. 